\section{Ethical Aspects of ES Cell-Derived Gametes}

\section{Giuseppe Testa* and John Harris}

$\mathbf{T}$ hree laboratories recently reported derivation of gamete-like cells from mouse embryonic stem (ES) cells (1-4). Much progress must be made to reach the goal of deriving bona fide germ cells from ES cells; the chief test will be their ability to sustain normal fertilization and development. However, these cells appear to share, to some degree, fundamental features of gametes, namely, a haploid genome and erasure of imprints (i.e., the molecular marks that distinguish alleles according to their origin and are essential for normal development).

The most immediate application of ESderived oocytes will likely be in somatic cell nuclear transfer (SCNT) (5) for research purposes. Clearly, many challenges remain before such gametes could be used in human reproduction. Precisely because the technology is not yet within immediate reach, we believe it is timely to start developing a bioethical and legal discourse.

The possibility of deriving gametes from human ES cells could allow infertile individuals to have genetic offspring, unless the defect also impaired in vitro generation of gametes. Infertility affects roughly 2.1 million couples in the United States alone (6). A somatic nucleus from the infertile member of the couple would be transplanted into an enucleated oocyte. ES cells would be derived from the blastocyst stage of this cloned embryo and differentiated into gametes, which would be used for in vitro fertilization (IVF) with the naturally generated gamete from the fertile partner. Although the procedure involves SCNT, this would not amount to reproductive cloning, because the offspring would have, as in normal reproduction and IVF, the genomic contribution of both parents. Simply, whereas the haploid genome from the fertile member of the couple will have been reprogrammed ("made fit for fertilization") through the normal process of gametogenesis, the infertile member will have

G. Testa is a Branco Weiss Society-in-Science Fellow in the Department of Genomics, BIOTEC, Dresden University of Technology, Dresden, Germany. E-mail: testa@mpi-cbg.de. J. Harris is the Sir David Alliance Professor of Bioethics at the Manchester School of Law, University of Manchester, Manchester, M13 9PL UK.

*Author for correspondence. achieved the same result with an "assisted reprogramming effort" mediated through the resetting activity of a donor oocyte. We suggest that from an ethical and legal perspective, this procedure is most appropriately framed as a therapeutic intervention to treat infertility. It replaces in vitro the physiologic function normally responsible for reprogramming the germline genome, analogously to the well-established medical technologies that replace other deficient bodily functions.

If the technique approaches the same level of safety and cost as those of current IVF protocols, it is likely that the majority of infertile individuals and/or couples would want to benefit from it. However, this does not imply any preeminence of genetic over social parenthood. Although our society values social parenthood highly, it still actively encourages those who can to have genetic offspring. Provided that the technology would be widely and justly accessible, this could further democratize human reproduction.

As with any medical procedure, safety would be a crucial parameter. Faulty imprinting causes severe abnormalities, and careful experiments in animal models will have to assess the imprinting status of ES-derived gametes (ESDG). Another potential problem could result from the accumulation of genetic damage that occurs in a somatic cell. However, ethical arguments based generally on DNA integrity would not be tenable if it were to be shown that oocytes or sperm from older individuals have amounts of damage similar to those of somatic lineages used for ESDG generation. As with IVF, evaluation of safety always entails a comparison with the safety of natural or other accepted processes rather than of ideal absolutes. The identification of the normal standard for any biological system is always a complex settlement between our incomplete knowledge and the risks we are prepared to take as individuals and as a society.

ESD oocytes have been derived from male $(\mathrm{XY})$ and female (XX) mouse cells (1). If the process could be successfully completed with human ES cells, i.e., derivation of a mature oocyte from a male ES cell line, this would have important social implications. Two men could potentially have a child to which both parents contribute their genomes, one through the natural process of spermatogenesis, the other through the assisted process of genome reprogramming down the female germline. A woman would need to carry this embryo to term. The possibility of an all-male or all-female couple's (7) being able to have a child sharing the genetic make-up of both parents in virtually the same way as for heterosexual couples is thought-provoking and can be used as a lens through which to discern our attitudes toward parenting and family, as well as our notions of what is "natural."

Same-sex couples can use assisted reproductive technologies (ART) in the United Kingdom to have children to whom they are at least in part genetically related and are able to adopt children in several countries. There have been extensive analyses of the success of same-sex couples as parents in the context of ART and adoption (8). As with most forceful rejections of social changes that violate presumed natural laws $(9,10)$, so for ESDGbased technology, most objections will likely arise from the idea that the resulting child will somehow be harmed by the nature, novelty, or "strangeness" of the process itself. This is analogous to the early days of IVF, when it was suggested that "test tube" babies might not have a soul (11). In our view, not only does a child born as a result of ART or ESDG obviously have the same moral status as a child born by natural reproduction, but would also have no special advantages or disadvantages. Natural reproduction may be more enjoyable for humans than the in vitro approaches, and that would certainly be a good reason to prefer it. However, there is no a priori reason to prefer the natural, for the natural per se is morally neutral. The whole practice of medicine is a comprehensive attempt to frustrate the course of nature. If we always preferred the natural as a matter of principle, we would have to abjure medicine altogether.

\section{References and Notes}

1. K. Hubner et al., Science 300, 1251 (2003).

2. Y. Toyooka, N. Tsunekawa, R. Akasu, T. Noce, Proc. Natl. Acad. Sci. U.S.A. 100, 11457 (2003).

3. N. Geijsen et al., Nature 427, 148 (2004).

4. C. Dennis, Nature 424, 364 (2003).

5. A. M. Surani, Nature 427, 106 (2004).

6. National Center for Health Statistics (NCHS), "Fertility, family planning and womens' health: New data From the 1995 National Survey of Family Growth" (NCHS, Hyattsville, MD, 1996).

7. T. Kono et al., Nature 428, 860 (2004).

8. S. Golombok, in What Is a Parent?: A Socio-Legal Analysis, A. Bainham, S. D. Sclater, M. Richards, Eds. (Hart, Oxford, 1999), pp. 161-180.

9. L. Kass, Valparaiso Univ Law Rev. 32, 679 (Spring 1998).

10. And for a refutation of such views, see J. Harris, On Cloning (Routledge, London, 2004), p. $52 \mathrm{ff}$.

11. See www.msnbc.msn.com/id/3990309/site/newsweek/

12. The authors acknowledge support from the Branco Weiss Fellowship Society-in-Science and a project grant from the European Commission. 